\title{
TOTALLY GEODESIC SURFACES IN HYPERBOLIC 3-MANIFOLDS
}

\author{
by ALAN W. REID \\ (Received 27th January 1989, revised 27th February 1990)
}

\begin{abstract}
In this paper we investigate totally geodesic surfaces in hyperbolic 3-manifolds. In particular we show that if $M$ is a compact arithmetic hyperbolic 3-manifold containing an immersion of a totally geodesic surface then it contains infinitely many commensurability classes of such surfaces. In addition we show for these $M$ that the Chern-Simons invariant is rational.

We also show, that unlike the figure-eight knot complement in $S^{3}$, many knot complements in $S^{3}$ do not contain an immersion of a closed totally geodesic surface.
\end{abstract}

1980 Mathematics subject classification (1985 Revision): Primary 57M99 Secondary 20H10.

\section{Introduction}

By a hyperbolic 3-manifold (resp. surface) we shall mean a complete, orientable 3-dimensional (resp. 2-dimensional) Riemannian manifold of constant curvature-1, of finite volume.

The conjectural picture of hyperbolic 3-manifolds as suggested by the work of Thurston and Waldhausen is that every such 3-manifold contains an immersed, incompressible surface (i.e. $\pi_{1}$ (surface) injects in $\pi_{1}$ (3-manifold)). The geometrically simplest situation that arises is when the immersion is totally geodesic. This has been of interest lately (see for example $[1,2,11,13,14,17]$ ) since for example it is shown in [11] that if a closed hyperbolic 3-manifold $M$ contains an immersion of a totally geodesic surface, then there is a finite cover $M_{0}$ of $M$ which contains an embedded, nonseparating totally geodesic surface (so in particular the first betti number of $M_{0}$ is positive).

Thus, it seems worthwhile to understand and classify those hyperbolic 3-manifolds which contain an immersed totally geodesic surface. Equivalently, via the holonomy representation of the fundamental groups, this means understanding the Fuchsian subgroup structure (where by a Fuchsian group we mean a discrete subgroup of $P S L_{2}(\mathbb{C})$ which fixes a circle or straight-line $\mathscr{C}$ in $\mathbb{C}$ and preserves components of $\mathbb{C} \backslash \mathscr{C}$ ) of the relevant Kleinian group.

In [14] we classified those arithmetic Kleinian groups (see Section 2 for a definition) which contain non-elementary Fuchsian groups. In [13] it was shown already that the Bianchi groups $P S L_{2}\left(O_{d}\right)$ (where $O_{d}$ is the ring of integers in $\mathbb{Q}(\sqrt{-d})$ ) all contain non-elementary Fuchsian subgroups. In fact it is shown in [13] that there exist both 
non-cocompact Fuchsian subgroups of finite covolume and cocompact Fuchsian subgroups and that each group $\operatorname{PS} L_{2}\left(O_{d}\right)$ contains infinitely many commensurability classes of cocompact (arithmetic) Fuchsian subgroup.

Our first main result is a generalization of this, namely:

Theorem 1. Let $\Gamma$ be a cocompact arithmetic Kleinian group. If $\Gamma$ contains a non-elementary Fuchsian subgroup then $\Gamma$ contains infinitely many commensurability classes (up to conjugacy in $P S L_{2}(\mathbb{C})$ ) of cocompact (necessarily arithmetic) Fuchsian subgroups.

As stated above, the groups $\operatorname{PSL}_{2}\left(O_{d}\right)$ all contain infinitely many commensurability classes of cocompact Fuchsian subgroups. Thus the same holds for any subgroup of finite index in $P S L_{2}\left(O_{d}\right)$. In particular the group of the figure-eight knot has an excellent representation (in the terminology of [23]) as a subgroup of index 12 in $P S L_{2}\left(O_{3}\right)$ (cf. [22]). In [18] this knot was shown to be the only arithmetic knot (see [18] for a definition). Our next main result is (in comparison with above).

Theorem 3. There exist infinitely many non-commensurable hyperbolic knot complements in $S^{3}$ which contain no closed totally geodesic surfaces and exactly one commensurability class of non-closed totally geodesic surface.

On a different theme, the Chern-Simons invariant (cf. [16] for a definition) is proving useful in distinguishing hyperbolic 3-manifolds. However, it is still a little mysterious with not much known about the values it can assume. Here we show in an elementary manner that the existence of an immersed totally geodesic surface in an arithmetic hyperbolic 3-manifold implies the Chern-Simons invariant is rational $\left(\bmod \frac{1}{2}\right)$.

\section{Arithmetic Kleinian groups and their Fuchsian subgroups}

2.1. Arithmetic Kleinian groups are obtained as follows (cf. [3] or [30, Chap. 4]). Let $k$ be a number field with one complex place and let $A$ be a quaternion algebra defined over $k$ such that $A \bigotimes_{k} k_{v}$, where $v$ is a real place of $k$, is isomorphic to the Hamiltonian quaternions over $\mathbb{R}$. Let $\rho$ be an isomorphism of $A$ into $M_{2}(\mathbb{C}), \mathcal{O}$ an order of $A$ and $\mathcal{O}^{1}$ the elements of $\mathcal{O}$ of norm 1. Then $P \rho\left(\mathcal{O}^{1}\right)$ (where $P: S L_{2}(\mathbb{C}) \rightarrow P S L_{2}(\mathbb{C})$ ) is a Kleinian group of finite covolume (cf. [30, Chap. 4]) and the class of arithmetic Kleinian groups is that given by the union of the commensurability classes of all such as $P \rho\left(\mathcal{O}^{1}\right)$. In addition we say that a Kleinian group $\Gamma$ is derived from a quaternion algebra if $\Gamma$ is contained in some $P \rho\left(\mathcal{O}^{1}\right) . H^{3} / \Gamma$ is arithmetic (resp. derived from a quaternion algebra) when $\Gamma$ is so.

Arithmetic Fuchsian groups (and Fuchsian groups derived from a quaternion algebra) are similarly obtained: in this case the field $k$ is totally real and the quaternion algebra is ramified at all the archimedian places except one which may be taken to be the identity. 
Notation. We now reserve the term "Fuchsian group" to mean non-elementary Fuchsian group.

2.2. The following two theorems were proved in [14] (and [17]).

Theorem M-R 1. Every Fuchsian subgroup of an arithmetic Kleinian group is a subgroup of an arithmetic Fuchsian group.

Theorem M-R 2. Let $\Gamma$ be an arithmetic Kleinian group with associated algebra $A$ defined over $k$. Then $\Gamma$ contains Fuchsian subgroups if and only if the following two conditions are satisfied:

1. $[k: l]=2$ where $l=k \cap \mathbb{R}$.

2. $A \cong B \bigotimes_{l} k$ where $B$ is a quaternion algebra over $l$ of a type giving arithmetic Fuchsian groups.

Remarks. 1. As stated in the introduction, if $\Gamma$ is a cocompact, torsion-free, arithmetic Kleinian group satisfying theorem MR-2 the manifold $H^{3} / \Gamma$ (where $H^{3}$ is hyperbolic 3-space) has a finite cover $M_{0}$ which contains an embedded non-separating closed totally geodesic surface. Thus $M_{0}$ has positive first betti number. In [6] arithmetic hyperbolic 3-manifolds $H^{3} / \Delta$ are constructed with positive first betti number such that $\Delta$ does not satisfy the conditions of Theorem M-R 2. In particular $H^{3} / \Delta$ contains an embedded incompressible surface which is not totally geodesic. Although this was to be expected it has proved difficult in practice to construct examples.

In a similar vein, in [7] it is shown that for all $d \neq 1,2,3,7,11$ the groups $P S L_{2}\left(O_{d}\right)$ contain, in addition to many Fuchsian subgroups, a subgroup isomorphic to a cocompact Fuchsian group containing an accidental parabolic element (so are not Fuchsian groups).

2.3. To prove Theorem 1 we shall reformulate Theorem $M-R 2$ in terms of the ramification sets of the quaternion algebras $A$ and $B$ of Theorem M-R 2 .

Firstly, we recall some elementary facts from the theory of prime ideals in a quadratic number field extension $K \mid L$.

Let $\not h$ be an $L$-prime; we say

(i) $\not h$ decomposes in $K \mid L$ if $\not 2 R_{K}=\mathscr{P P P ^ { \prime }}$ for $K$-primes $\mathscr{P}, \mathscr{P}^{\prime}$ with $K_{\mathscr{P}} \cong K_{\mathscr{P}} \cong L_{\not}$.

(ii) $\not h$ ramifies in $K \mid L$ if $\not 2 R_{K}=\mathscr{P}^{2}$ for a $K$-prime $\mathscr{P}$ with $K_{\mathscr{P}} \cong L_{\not p}$.

(iii) $\not h$ inert in $K \mid L$ if $\not h R_{K}=\mathscr{P}$ for a $K$-prime $\mathscr{P}$ with $\left[K_{\mathscr{P}}: L_{\AA}\right]=2$.

where $L_{f}, K_{\mathscr{P}}$ and $K_{\mathscr{P}}$. denote the completions of the fields at the place corresponding to the primes at the $\not h, \mathscr{P}$ and $\mathscr{P}^{\prime}$ respectively.

More precisely, we have natural inclusions $i: L \rightarrow K$ (resp. $j_{\not}: L \rightarrow L_{\star}$ and $j_{\mathscr{g}}: K \rightarrow K_{\mathscr{P}}$ ) which induce a map $\hat{i}$ such that the following diagram comments: 


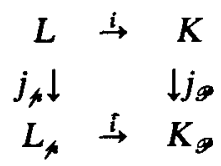

In case (i), $\hat{i}$ is the indicated isomorphism, however in case (ii) we have (in the above notation):

Lemma 1. $\left[K_{g}: \hat{i}\left(L_{\not p}\right)\right]=2$.

Proof. Let $\pi_{f}$ (resp. $\pi_{\mathscr{P}}$ ) be a local uniformizing parameter for $L_{f}$ (resp. $K_{\mathscr{P}}$ ). The lemma follows form the fact that $\hat{i}\left(\pi_{k}\right)=\pi_{\mathscr{P}}^{2}$.

Theorem 1 is a consequence of the next proposition for which we require some additional notation.

Notation. Let $A$ be a quaternion algebra over the number field $K$. A place $v$ of $K$ (archimedean or non-archimedean) is said to ramify $A$ if $A \bigotimes_{K} K_{v}$ is a division algebra of quaternions. In particular if $v$ is archimedean and $v$ ramifies $A$ then $v$ is necessarily real since there is only one quaternion algebra over $\mathbb{C}$ up to isomorphism, namely $M_{2}(\mathbb{C})$.

The set of places which ramify $A$ is of finite even cardinality, and we denote the subset of non-archimedean places ramifying $A$ by $\operatorname{Ram}_{f} A$ (cf. [30, Chap. 3] for details).

Proposition 1. Let $A$ be a division algebra of quaternions over the field $k$ with one complex place of the type described in Section 2.1. Let $l=k \cap \mathbb{R}$ and $[k: l]=2$. Let $B$ be $a$ quaternion algebra over l ramified at all real places except the identity. Then $A \cong B \bigotimes_{l} k$ if and only if $\operatorname{Ram}_{f} A=\emptyset$ or $\operatorname{Ram}_{f} A=\left\{\mathscr{P}_{1}, \mathscr{P}_{1}^{\prime}, \ldots, \mathscr{P}_{r}, \mathscr{P}_{r}^{\prime}\right\}$ where $\mathscr{P}_{i} \cap R_{l}=\mathscr{P}_{i}^{\prime} \cap R_{l}=p_{i}$ for $i=1, \ldots, r\left(R_{l}\right.$ denotes the ring of integers of $\left.l\right)$ and either $\operatorname{Ram}_{f} B=\emptyset$ of $\operatorname{Ram}_{f} B \supseteq$ $\left\{\mathfrak{h}_{1}, \ldots, \mathfrak{h}_{r}\right\}$ and $\operatorname{Ram}_{f} B \backslash\left\{\mathfrak{h}_{1}, \ldots, \mathfrak{h}_{r}\right\}$ consists of l-primes which are ramified or inert in $k \mid l$.

Proof. As $[k: \mathbb{Q}]$ is even we have from the remark prior to Proposition 1 , the possibility that the ramification set of $A$ consists of only real archimedean places i.e. $\operatorname{Ram}_{f} A=\emptyset$. Otherwise $\operatorname{Ram}_{f} A$ is of even cardinality. To describe the relationship between $\operatorname{Ram}_{f} A$ and $\operatorname{Ram}_{f} B$ we use the following lemma which follows from basic properties of tensor product (a proof is given in [17]).

Lemma 2. Under the natural maps $i, \hat{i}, j_{k}$ and $j_{g}$ above the tensor products $\left(B \bigotimes_{l} k\right) \bigotimes_{k} k_{g}$ and $\left(B \bigotimes_{l} l_{k}\right) \bigotimes_{l_{\sharp}} k_{g}$ are isomorphic.

Thus suppose that $A \cong B \bigotimes_{l} k$ and $\mathscr{P} \in \operatorname{Ram}_{f} A$. To deduce the structure of $\operatorname{Ram}_{f} B$ we apply Lemma 2.

Firstly, let us assume that $\not h=\mathscr{P} \cap R_{l}$ is inert in $k \mid l$. By Lemma $2 B$ is ramified at $\not h$. Hence 


$$
\begin{aligned}
A \bigotimes_{k} k_{g} & \cong\left(B \bigotimes_{1} k\right) \bigotimes_{k} k_{k} \quad \text { by hypothesis } \\
& \cong\left(B \bigotimes_{1} l_{k}\right) \bigotimes_{l} k_{\sharp} \quad \text { by Lemma } 2 \\
& \cong D_{k} \bigotimes_{l_{k} k_{g}}
\end{aligned}
$$

where $D_{k}$ is the unique division algebra of quaternions (up to isomorphism) over $l_{k}$ (cf. [30, Chap. 2]).

Now $\left[k_{g}: l_{\sharp}\right]=2$ and it is known (cf. [30, Chap. 2, Theorem 1.3]) that any quadratic extension of $l_{\mathscr{A}}$ splits $D_{\mathscr{P}}$. But $A$ is ramified at $\mathscr{P}$ and hence a contradiction. Thus if $\mathscr{P} \in \operatorname{Ram}_{f} A, \mathscr{P} \cap R_{l}$ is not inert in $k \mid l$. Similarly using Lemma 1 we can eliminate $k$-primes $\mathscr{P}$ such that $\mathscr{P} \cap R_{l}$ is ramified in $k \mid l$ from $\operatorname{Ram}_{f} A$.

Consequently if $\operatorname{Ram}_{f} A \neq \emptyset$, it can only contain primes $\mathscr{P}, \mathscr{P}^{\prime}$ such that $\mathscr{P} \cap R_{l}=$ $\mathscr{P} \cap \cap \boldsymbol{R}_{l}$. It also easily follows from Lemma 2 that if $A$ is ramified at $\mathscr{P}$ then $A$ is ramified at $\mathscr{P}^{\prime}$. Thus we have proved one half of Proposition 1.

For the converse we must construct the relevant quaternion algebra $A$. This depends on an easy case-by-case construction depending on $r$ and $[l: \mathbb{Q}] \bmod 2$ (cf. [17] for more details).

Remark. From the classification theorem for quaternion algebras it is known that for every finite set $S$ of places of a number field $k$ such that $|S|$ is even, there exists a quaternion algebra over $k$ with $S$ as its ramifying set. Restricting to fields $k$ with 1 complex place and sets $S$ which contain all real infinite places of $k$, we see from Theorem M-R 2 and Proposition 1 that in general an arithmetic hyperbolic 3-manifold will not contain an immersion of a totally geodesic surface. This is indicative of what is expected in the general situation; that is, incompressible surfaces in hyperbolic 3manifolds are not totally geodesic. On the other hand, until the results of [14], explicit examples of hyperbolic 3-manifolds with no totally geodesic surfaces had proved difficult to construct.

Proof of Theorem 1. By Theorem M-R 1, if $\Gamma$ contains a non-elementary Fuchsian subgroup, it will certainly contain an arithmetic Fuchsian subgroup, say $F$, with associated quaternion algebra $B$ defined over $l$. By Theorem M-R 2 it follows that the ramification sets of the quaternion algebras $A$ (that associated to $\Gamma$ ) and $B$ are related by Proposition 1 .

To produce infinitely many commensurability classes (up to conjugacy in $P S L_{2}(\mathbb{C})$ ) of arithmetic Fuchsian subgroup of $\Gamma$, it suffices (by Proposition 1 of [28]) to exhibit infinitely many isomorphism classes of quaternion algebras $\hat{B}$ defined over $l$ such that $\hat{B} \otimes_{1} k \cong A$. From the theory of quaternion algebras this will be determined by distinct sets of finite primes of $l$. These we produce using the above $B$.

By Proposition 1, $\operatorname{Ram}_{f} B=\emptyset$ or $\operatorname{Ram}_{f} B \supseteq\left\{h_{1}, \ldots, h_{r}\right\}$ with notation as in Proposition 1. Let $k, k^{\prime}$ be distinct primes of $l$ which are inert in $k \mid l$ (infinitely many such primes 
exist, as $k \mid l$ is of degree 2; see for instance [8, Chap. 7, Corollary 9.2.7]) not lying in $\operatorname{Ram}_{f} B$.

Define the algebra $B\left(\not, \not^{\prime}\right)$ over $l$ to be that which is ramified at all real places except the identity and $\operatorname{Ram}_{f} B\left(\not h, p^{\prime}\right)=\operatorname{Ram}_{f} B \cup\left\{\not, \not^{\prime}\right\}$ (this is guaranteed by the classification theorem for quaternion algebras).

By Theorem M-R 2 and Lemma $2 B\left(\not h, \mathfrak{h}^{\prime}\right)$ gives arithmetic Fuchsian subgroups of $\Gamma$. The theorem now follows from our remark that infinitely many $\not h, \mathfrak{h}^{\prime}$ exists.

We have, when $\Gamma$ is torsion-free:

Corollary 1. The arithmetic hyperbolic 3-manifold $H^{3} / \Gamma$ contains infinitely many incommensurable immersed closed totally geodesic surfaces.

\section{The Chern-Simons invariant}

3.1. Let $M$ be a hyperbolic 3-manifold, and let $C S(M)$ denote the Chern-Simons invariant of $M$ (cf. [16] for a definition and more details). Now $C S(M)$ takes values in the circle $\mathbb{R} / \mathbb{Z}$ (in fact in $[16]$ it is shown how to compute $C S(M) \bmod \frac{1}{2}$ ); however the values which it can assume are somewhat mysterious and their computations difficult.

However, for those arithmetic hyperbolic 3-manifolds which contain an immersion of a totally geodesic surface we can prove:

Theorem 2. Let $M=H^{3} / \Gamma$ where $\Gamma$ is an arithmetic Kleinian group which contains Fuchsian subgroups. Then $C S(M)$ is rational $\left(\bmod \frac{1}{2}\right)$.

Proof. The proof of Theorem 2 will follow from Lemma 3 below (which was noticed in [17]) and elementary properties of the Chern-Simons invariant.

Lemma 3. Let $M$ be as in the statement of Theorem 2. Then there exists a manifold $M_{0}$ commensurable with $M$ which admits an orientation-reversing involution.

Proof. There is no loss in assuming that $\Gamma \subset \rho\left(\mathcal{O}^{1}\right)$ where $\mathcal{O}$ is an order of the associated quaternion algebra of $\Gamma$ (which for the sake of notation we will assume is a discrete subgroup of $S L_{2}(\mathbb{C})$ ) and $\rho$ is as in Section 2.1. It follows from Theorem M-R 2 (cf. [14, Theorem 8] for details) that we can assume the associated quaternion algebra of $\Gamma$ has the form $A=(a, b / k)$ where $a, b$ are elements of the field $l=k \cap \mathbb{R}$.

Let $\sigma$ denote the non-trivial galois monomorphism of $k \mid l$, so that $\sigma$ is just complex conjugation. Clearly $\sigma$ extends to $A$ and from above we get $\sigma(A)=(\sigma(a), \sigma(b) / \sigma(k))=(a, b /$ $k)=A$. Thus $\sigma$ is an automorphism of $A$, hence by the Skölem-Noether theorem there exists $\delta \in A^{*}$ such that $\sigma(\alpha)=\delta \alpha \delta^{-1}$.

Now $\sigma(\rho(\mathcal{O}))$ is again an order of $\rho(A)$ so that $\sigma(\Gamma)=\delta \Gamma \delta^{-1}$ is commensurable with $\Gamma$. Let $\Delta=\Gamma \cap \delta \Gamma \delta^{-1}$ and $M_{0}=H^{3} / P \Delta$ where $\left(P: G L_{2}(\mathbb{C}) \rightarrow P G L_{2}(\mathbb{C})\right)$. It now follows that $M_{0}$ admits an orientation-reversing involution. 
The proof of Theorem 2 is then a consequence of Lemma 3 and the following two facts about the Chern-Simons invariant noted in Section 2.2 of [16]:

(i) reversing the orientation of $M$ changes the sign of $C S(M)$.

(ii) taking the $n$-fold cover of a manifold multiplies $C S(M)$ by a factor of $n$.

Remark. It might be tempting to conjecture that $C S(M)$ is rational for any arithmetic, hyperbolic 3-manifold $M$. However, using computer calculations of Walter Neumann, one can construct arithmetic hyperbolic 3-manifolds $M$ for which $\operatorname{CS}(M)$ is "seemingly" irrational. One such example is $(5,1)$ surgery on the complement of the figure eight-knot. This is known to be arithmetic by [5].

3.2. It is well-known from the results of Borel [3] that if we fix a field $k$ with one complex place and consider arithmetic Kleinian groups arising from quaternion algebras over $k$ then the covolumes of these groups are rationally related even though the groups themselves may be incommensurable. From Theorem 2 we have:

Corollary 2. The set of Chern-Simons invariants of arithmetic hyperbolic 3-manifolds which contain an immersion of a totally geodesic surface are all rationally related.

\section{Totally geodesic surfaces in knot complements}

4.1. As remarked in the introduction, the complement of the figure-eight knot in $S^{3}$ contains many closed totally geodesic surfaces-which by [9] cannot be embedded (see [15] where the minimal genus of such a surface is computed). Thus, a natural question is to ask whether this phenomena occurs more generally among knot complements; particularly 2-bridge knot complements. Here we prove:

Theorem 3. There exist infinitely many non-commensurable hyperbolic knot complements in $S^{3}$ which contain no closed totally geodesic surfaces and exactly one commensurability class of non-closed totally geodesic surface.

The proof of Theorem 3 will be a consequence of our next proposition and the work of Riley [20] and [21].

4.2. In this section we aim to prove:

Proposition 2. Let $\Gamma$ be a non-cocompact Kleinian group of finite covolume such that:

(i) $k=\mathbb{Q}(t r \gamma: \gamma \in \Gamma)$ contains no proper real subfield other than $\mathbb{Q}$ and $[k: \mathbb{Q}]$ is odd.

(ii) $\operatorname{tr} \Gamma=\{\operatorname{tr} \gamma: \gamma \in \Gamma\} \subset R_{k}$-the ring of integers of $k$.

Then $\Gamma$ contains no cocompact Fuchsian subgroups and at most one commensurability class (up to conjugacy in $P S L_{2}(\mathbb{C})$ ) of non-cocompact Fuchsian subgroup of finite covolume. 
Proof. Firstly I claim that if $\Gamma$ contains Fuchsian subgroups of finite covolume then they are Fuchsian groups derived from a quaternion algebra (recall Section 2.1).

Thus, let $F \subset \Gamma$ be a Fuchsian subgroup of finite covolume. As the traces of the elements of $F$ are real we have by (ii) that $\operatorname{tr} F \subset R_{k} \cap \mathbb{R}$ and $R_{k} \cap \mathbb{R}=\mathbb{Z}$ by (i). However, the main result of [25] implies that integer traces characterizes a Fuchsian group derived from a quaternion algebra defined over $\mathbb{Q}$. This proves the claim asserted above.

Now let $A(F)=\left\{\sum_{i} a_{i} f_{i}: a_{i} \in \mathbb{Q}, f_{i} \in F\right\}$ and $A(\Gamma)=\left\{\sum_{i} b_{i} \gamma_{i}: b_{i} \in k, \gamma_{i} \in \Gamma\right\}$. Then $A(F)$ and $A(\Gamma)$ are quaternion algebras over $\mathbb{Q}$ and $k$ respectively (cf. [25, Proposition 3] for example). Moreover, clearly $A(F)$ is a subalgebra of $A(\Gamma)$.

Lemma 4. $A(\Gamma) \cong M_{2}(k)$.

Proof. Since $\Gamma$ contains parabolic elements, this implies that $A(\Gamma)$ contains zero divisors and hence as $A(\Gamma)$ is a quaternion algebra over $k$ the lemma follows.

Now a dimension count and Lemma 4 shows that the quaternion algebra $A(F) \bigotimes_{\mathrm{Q}} k$ is isomorphic to $M_{2}(k)$.

We can now complete the proof of Proposition 2. Suppose $F$ is cocompact, in which case $A(F)$ is ramified at some prime $p \in \mathbb{Z}$. Let $h_{1}, \ldots, h_{g}$ be the $k$-prime divisors of $p$. Now Lemma 2 can be suitably modified to yield in this case:

$$
\left(A(F) \bigotimes_{\mathbb{Q}} k\right) \bigotimes_{k} k_{p_{i}} \cong\left(A(F) \bigotimes_{\mathbb{Q}} \mathbb{Q}_{p}\right) \bigotimes_{\mathrm{Q}_{p}} k_{p_{i}}
$$

for each $k$-prime divisor $\mathfrak{h}_{i}, i=1, \ldots, g$.

But the left-hand side of (I) is $M_{2}\left(k_{k_{i}}\right)$ from Lemma 4 and the classification theorem for quaternion algebras ([30, Chap. 3]). Thus

$$
M_{2}\left(k_{p_{i}}\right) \simeq\left(A(F) \bigotimes_{0} \mathbb{Q}_{p}\right) \bigotimes_{\mathbf{Q}_{p}} k_{k_{i}} \text { for } i=1, \ldots, g .
$$

As $A(F)$ is ramified at $p, A(F) \bigotimes_{\mathbb{Q}} \mathbb{Q}_{p}$ is (isomorphic to) the unique division algebra of quaternions over $\mathbb{Q}_{p}$ (cf. [30, Chap. 2]). However this algebra is split by a field $K$ if and only if $\left[K: \mathbb{Q}_{p}\right]$ is even (cf. [30, Chap. 2, Theorem 1.3]). Thus $n_{i}=\left[k_{p_{i}}: \mathbb{Q}_{p}\right]$ is even for each $i=1, \ldots, g$. It is standard fact from local-global principles in number theory that $[k: \mathbb{Q}]=\sum_{i=1}^{g} n_{i}$ (see for example [8, Corollary 5.1.7]). But $[k: \mathbb{Q}]$ is odd by assumption and from above $n_{i}$ is even for each $i=1, \ldots, g$. This contradiction implies no cocompact Fuchsian subgroups.

Moreover, since up to conjugacy in $P S L_{2}(\mathbb{C})$ there is only one commensurability class of non-cocompact arithmetic Fuchsian group, Proposition 2 now follows.

Remark. A nice consequence of Lemma 4 is that the fundamental group of any non-compact hyperbolic 3-manifold $M$ has a faithful discrete representation $\Gamma$ such that $\Gamma \subset P S L_{2}(\mathbb{Q}(\operatorname{tr} \Gamma))$. To see this, we can conjugate a faithful discrete representation of $\pi_{1}(M)$ in $P S L_{2}(\mathbb{C})$ to contain the elements $T_{1}=\left(\begin{array}{ll}1 & 1 \\ 0 & 1\end{array}\right)$ and $T_{2}=\left(\begin{array}{ll}1 & 0 \\ \alpha & 1\end{array}\right)$ for some $\alpha \in \mathbb{C}, \alpha \neq 0$. 


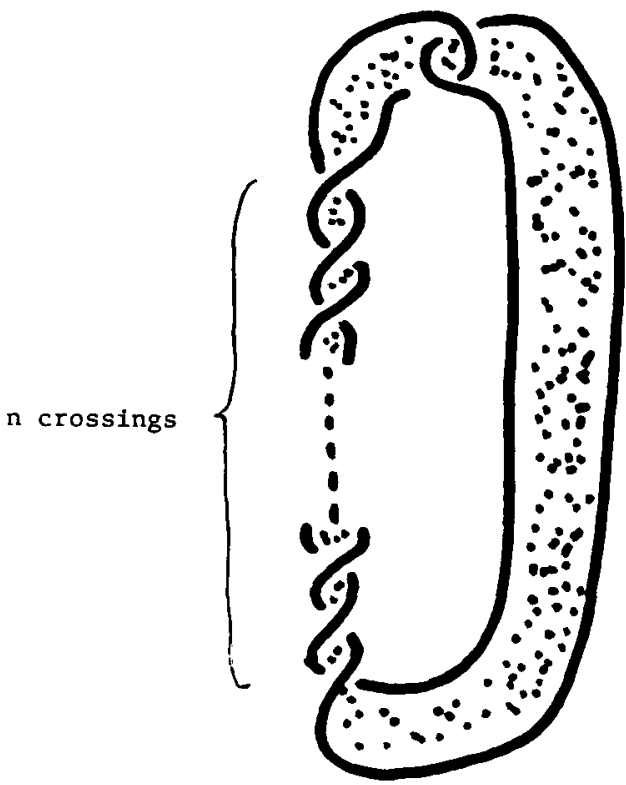

FIGURE 1

Notice that $\operatorname{tr}\left(T_{1} T_{2}\right)=2+\alpha$, so that $\alpha \in \mathbb{Q}(\operatorname{tr} \Gamma)$. But then Proposition 3.1 of [12] implies the coefficient field of this faithful discrete representation coincides with $\mathbb{Q}(\operatorname{tr} \Gamma)$.

4.3. In this section we shall prove Theorem 3, which will require work of Riley on 2-bridge knots, relevant details of which are reviewed below (see [4, Chap. 12] or [20] for details on 2-bridge knots).

Now a twist knot $K$ (see Figure 1) is a 2-bridge knot with 2-bridge normal form $(\alpha, \alpha-2)$ for some $\alpha \in \mathbb{Z}$. The degree of the representative polynomial $\Lambda_{\alpha}(y)$ of $K$ (cf. [20]) is $\lambda=\frac{1}{2}(\alpha-1)$. The following lemma is implicit in [21] (the notation is that of [23]):

Lemma 5. There exist infinitely many twist knots $K$ such that an excellent representation of $\pi_{1}\left(S^{3} \backslash K\right)$ satisfies (i) of Proposition 2.

Proof. Choose $\alpha$ so that $\alpha$ and $\lambda$ are both prime (from [21, Lemma 3] $\alpha$ is necessarily of the form $4 m+3)$. It now suffices to show that under these hypothesis $\Lambda_{\alpha}(y)$ is irreducible. However, this follows from [20]; in particular, the proof of Theorem 3 and Theorem 6 of [20] where it is shown that $\Lambda_{\alpha}(y)$ is irreducible modulo 2. As infinitely many such $\alpha$ exist the lemma follows.

We can now prove Theorem 3, since from Theorem 2 of [20] the traces of an excellent representation of any hyperbolic 2-bridge knot group satisfies (ii) of Proposi- 
tion 2. Thus we have constructed (from Lemma 5) infinitely many twist knot groups satisfying (i) and (ii) of Proposition 2. The final assertion is that twist knot complements given by Lemma 5 are all incommensurable, and this follows from Corollary 2 of [19] where it is shown that the trace-field of an excellent representation of a hyperbolic knot group is an invariant of the commensurability class.

Remarks. 1. Notice from Figure 1 we see (shaded region) an immersed twice punctured disc which is a thrice punctured sphere. Now any thrice punctured sphere is totally geodesic since the group of a thrice punctured sphere is the parabolic triangle group of type $(\infty, \infty, \infty)$ and all such are Fuchsian and conjugate in $P S L_{2}(\mathbb{C})$ (cf. [10, Lemma 4] or [1]). It also arithmetic by [27] as predicted by the proof of Proposition 2. Of course the figure-eight knot is a twist knot, thus it would be of interest to try to understand geometrically why there is this difference in the structure of the totally geodesic surfaces that occur in these knot complements.

2. From [23] and [24] together with unpublished work of Riley, the following 3bridge knots give further examples of knot complements with no closed totally geodesic surfaces:

$$
8_{10}, 8_{15}, 9_{22}, 9_{32}, 9_{35}, 9_{42}, 9_{48}
$$

3. Using the methods of $[1,2]$ and [29, Chap. 6], it is rather easy to construct many hyperbolic link complements which contain thrice punctured spheres. Moreover, by being careful in these constructions one can construct numerous non-arithmetic link complements containing thrice punctured sphere.

Examples of cocompact, non-arithmetic Kleinian groups which contain arithmetic Fuchsian subgroups can also be constructed (cf. [17]).

Acknowledgements. Part of this research was conducted during my $\mathrm{Ph} . \mathrm{D}$ at the University of Aberdeen and was funded by an S.E.R.C. grant which I gratefully acknowledge. I would also like to thank Colin Maclachlan and Walter Neumann for numerous helpful discussions and Robert Riley for making many of his unpublished computations available to me and to the referee for several instructive suggestions.

\section{REFERENCES}

1. C. C. Adams, Thrice punctured spheres in hyperbolic 3-manifolds, Trans. Amer. Math. Soc. 287 (1985), 645-656.

2. C. C. Adams, Augmented alternating link complements are hyperbolic, in Low-dimensional Topology and Kleinian groups (ed. D. B. A. Epstein, LMS Lecture Note Series 112 (1986), 115-130.

3. A. Borel, Commensurability classes and volumes of hyperbolic 3-manifolds, Ann. Scuola Norm. Sup. Pisa 8 (1981), 1-33.

4. G. Burde and H. Zieschang, Knots (De Gruyter Studies in Math. 5. Walter De Gruyter, Berlin, New York, 1985). 
5. T. Chinburg, A small arithmetic hyperbolic 3-manifold. Proc. Amer. Math. Soc. 100 (1987), 140-144.

6. L. Clozel, On the cuspidal cohomology of arithmetic subgroups of $S L(2 n)$ and the first betti number of arithmetic 3-manifolds, Duke Math. J. 55 (1987), 475-486.

7. C. Frohman and B. Fine, Some amalgam structures for Bianchi groups, Proc. Amer. Math. Soc. 102 (1988), 221-229.

8. L. J. Goldstein, Analytic Number Theory (Prentice-Hall Inc., New Jersey, 1971).

9. C. M. Gordon and R. A. LitherLand, Incompressible surfaces in branched coverings, in The Smith Conjecture (eds. J. W. Morgan and H. Bass, Academic Press, 1984). 139-152.

10. L. GreengerG, On a theorem of Ahlfors and conjugate subgroups of Kleinian groups, Amer. J. Math. 89 (1967), 55-68.

11. D. D. Long, Immersions and embeddings of totally geodesic surfaces, Bull. London Math. Soc. 19 (1987), 481-484.

12. A. M. MaCbeath, Commensurability of cocompact three dimensional hyperbolic groups, Duke Math. J. 50 (1983), 1245-1253.

13. C. Maclachlan, Fuchsian subgroups of the groups $P S L_{2}\left(O_{d}\right)$, in Low-dimensional Topology and Kleinian groups, ed. D. B. A. Epstein, L. M. S. Lecture Note Series 112 (1986), 305-311.

14. C. Maclachlan and A. W. Reid, Commensurability classes of arithmetic Kleinian groups and their Fuchsian subgroups, Math. Proc. Cambridge Philos. Soc. 102 (1987), 251-257.

15. C. Maclachlan and A. W. Reid, In preparation.

16. R. MeYerhoff, Density of the Chern-Simons' invaritant for hyperbolic 3-manifolds, in Low-dimensional Topology and Kleinian groups ed. D. B. A. Epstein, L. M. S. Lecture Note Series 112 (1986), 217-239.

17. A. W. ReID, Ph.d thesis, University of Aberdeen, 1987.

18. A. W. Reid, Arithmeticity of knot complements, J. London Math. Soc., to appear.

19. A. W. Reid, A note on trace-fields of Kleinian groups, Bull. London Math. Soc., to appear.

20. R. Riley, Parabolic representations of knot groups, I, Proc. London Math. Soc. (3)24 (1972), 217-247.

21. R. Riley, Knots with parabolic property P, Quart. J. Math. Oxford (2)25 (1974), 273-283.

22. R. Riley, A quadratic parabolic group, Math. Proc. Cambridge Philos. Soc. 77 (1975), 281-288.

23. R. Riley, Seven excellent knots, in Low-dimensional topology (eds. R. Brown and T. L. Thickstun, L. M. S. Lecture Note Series 48 (1982), 81-151.

24. R. RiLeY, Parabolic representations and symmetries of the knot $9_{32}$, in Computers in Geometry and Topology (ed. M. C. Tangora, Lecture notes in Pure and Applied Math. 114 (1988), 297-313.

25. K. TAKeUCH, On some discrete subgroups of $S L_{2}($ R). J. Fac. Sci. Univ. Tokyo Sect. IA Math. 16 (1969), 97-100.

26. K. TAKeuchI, A characterization of arithmetic Fuchsian groups. J. Math. Soc. Japan 27 (1975), 600-612.

27. K. TAKeUCHI, Arithmetic triangle groups, J. Math. Soc. Japan 29 (1977), 91-106. 
28. K. Takeuchl, Commensurability classes of arithmetic triangle groups, J. Fac. Sci. Univ. Tokyo Sect. IA Math. 24 (1977), 201-212.

29. W. P. Thurston, The geometry and topology of 3-manifolds (Mimeographed lecture notes, Princeton Univ., 1978).

30. Vigneras, M.-F., Arithmétique des algèbres de quaternions (Lecture Notes in Mathematics 800, Springer-Verlag, 1980).

31. N. J. Wielenberg, The structure of certain subgroups of the Picard group, Math. Proc. Cambridge Philos. Soc. 84 (1978), 427-436.

Department of Mathematics

The Ohio State University

231 West 18th Avenue

Columbus, OH 43210

USA 